\title{
OPTIC DISC DETECTION IN RETINAL IMAGES BY PATTERN DISTANCE MINIMIZATION
}

\author{
Marcy A. Dias and Fernando C. Monteiro \\ Polytechnic Institute of Bragança, Campus Santa Apolónia, Apartado 1134, 5301-857 Bragança, Portugal
}

\begin{abstract}
Keywords: Earth Mover's Distance, Gabor Wavelet Transform, Optic Disc Detection, Retinal Images.
Abstract: The retinal fundus photograph is widely used in the diagnosis and treatment of various eye diseases such as diabetic retinopathy and glaucoma. On the research work leading to automatic analysis of retinal images, the knowledge of the optic disc (OD) location is essential, and a new method to locate the optic disc automatically is proposed. We propose an algorithm for the detection of OD in the retina which takes advantage of the powerful preprocessing techniques such as the contrast enhancement, Gabor wavelet transform, mathematical morphology and Earth Mover's distance as the matching process. Forty images of the retina from the DRIVE database were used to evaluate the performance of the method.
\end{abstract}

\section{INTRODUCTION}

The retina is the inner-most layer of the eye where the earliest pathological changes are seen. It is composed of various anatomical structures which indicate many diseases, such as hypertension, diabetic retinopathy and glaucoma. Retinopathy is one of the main causes of blindness in the working age population. Analysis of retinal images is considered essential for diagnosis and treatment of many diseases affecting the retina. These images must be accurately segmented to extract sensitive objects in the retina such as the blood vessel tree, the optic disc, the macula and the region between the optic disc and the macula.

Retinal or fundus images provide information about the blood supply system to the retina. The optic disc is a bright area within a retinal image and is the exit point of retinal nerve fibers from the eye and the entrance and exit point for retinal blood vessels (See Fig. 1(a)). Optic Disc detection is a reference to locate the various anatomical features in the retinal images.

Several OD detection techniques make use of anatomical structures among the OD, macula, and retinal blood vessels (Youssif et al., 2008). For example, some methods are based on the anatomical structure that all major retinal blood vessels radiate from the OD. Some other methods make use of the relative position between the OD and the macula that often varies within a small range. Compared with the image characteristics, the anatomical structures are more reliable under the presence of retinal lesion and imaging artifacts. However, the extraction of either retinal blood vessels or the macula is often a nontrivial task by itself.

This paper presents a automatic method for optic disc detection which takes advantage of the powerful preprocessing techniques such as the contrast enhancement, Gabor wavelet transform, mathematical morphology and Earth Mover's distance for matching pattern. The methods include the design of a bank of directionally sensitive Gabor filters for several values of the scale and elongation parameters as proposed by Soares et al. (Soares et al., 2006). Forty images of the retina from the DRIVE database (Staal et al., 2004) were used to evaluate the performance of the method.

The rest of this paper is organized as follows. Section 2 describes the proposed OD detection technique. Experimental results are then described and discussed in Section 3. Some concluding remarks are finally drawn in Section 4.

\section{MATERIALS AND METHODS}

The DRIVE database consists of 40 images (seven of which present pathology), compressed in JPEG format of size $565 \times 584$ pixels, eight bits per colour channel, obtained from a diabetic retinopathy screening program. The images are acquired using a Canon CR5 nonmydriatic 3CCD camera at $45^{\circ}$ field of view. 
The 40 images were divided into two sets, a test set and a training set, each containing 20 images. The images have been manually segmented by three observers to be used as references for comparing the computer-generated segmentations.

\subsection{Contrast Adjustment}

After converting each pixel in the image to a vector of colour components and normalizing each component (dividing by 255), the result was converted to the luminance component $\mathrm{Y}$, computed as $Y=$ $0.299 R+0.587 G+0.114 B$, where $\mathrm{R}, \mathrm{G}$, and $\mathrm{B}$ are the red, green, and blue components, respectively, of the colour image. Several other works on the detection of retinal vessels have used the green channel only; in the present work, the $\mathrm{Y}$ channel was used in order to reduce noise by averaging the three colour component images and obtain an image with positive contrast for the vessels. The artifacts present in the DRIVE images at the edges were removed by applying morphological erosion with a disc-shaped structuring element of diameter 10 pixels.

As the contrast between the blood vessels (foreground) and the retinal tissue (background) is generally poor in the retinal images, an effective technique called contrast-limited adaptive histogram equalization (CLAHE) is utilized for contrast enhancement by limiting the maximum slope in the transformation function. Instead of applying the histogram equalization on the entire image, it is applied only on small non-overlapping regions in the image. Then, the neighboring tiles are combined using bilinear interpolation to reduce induced boundaries. Figure 1(b) shows the contrast enhancement produced by CLAHE approach.

\subsection{Blood Vessel Segmentation}

In many applications of image processing in ophthalmology, the most important step is to detect the blood vessels in the retina (Hoover et al., 2000),(Foracchia et al., 2004). In our case, we decided to choose the Soares et al. method (Soares et al., 2006) due to his high performance in blood vessel segmentation $(\approx 96 \%)$. The method produces segmentations by classifying each image pixel as vessel or nonvessel, based on the pixel's feature vector. Feature vectors are composed of the pixel's intensity and twodimensional Gabor wavelet transform responses taken at multiple scales. Gabor wavelets are sinusoidally modulated Gaussian functions that have optimal localization in both the frequency and space domains, thus allowing noise filtering and vessel enhancement

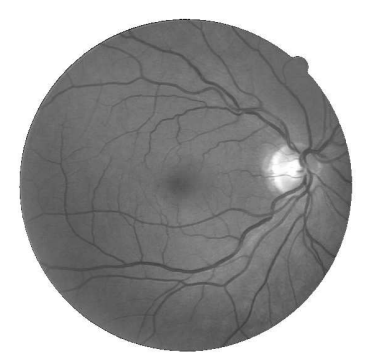

(a)

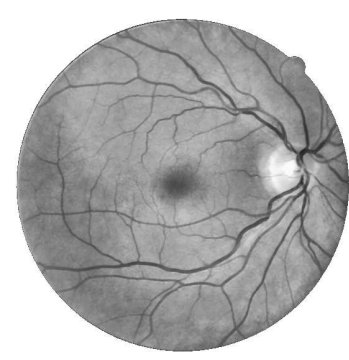

(b)
Figure 1: (a) Green channel of image, (b) Result of contrast enhancement with CLAHE.

in a single step. The wavelet is capable of detecting directional structures and of being tuned to specific frequencies, which is specially important for filtering out the background noise present in retinal images.

The 2-D Gabor wavelet is defined as

$$
\psi_{G}(\mathbf{x})=\exp \left(j \mathbf{k}_{\mathbf{0}} \mathbf{x}\right) \exp \left(-0.5|\mathbf{A} \mathbf{x}|^{2}\right)
$$

where $\mathbf{A}=\operatorname{diag}[1 / \sqrt{\varepsilon}, 1]$ is a $2 \times 2$ diagonal matrix that defines the anisotropy of the filter, i.e., its elongation in any desired direction. The Gabor wavelet is actually a complex exponential modulated Gaussian, where $\mathbf{k}_{\mathbf{0}}$ is a vector that defines the frequency of the complex exponential.

The Gabor wavelet transform is computed for spanning from 0 up to 170 degrees at steps of $10 \mathrm{de}$ grees. The maximum moduli of the wavelet transform over all angles for various scales are then taken as pixel features (Fig. 2(a)). In the tests performed, the elongation parameter was set to $\varepsilon=4$ and $\mathbf{k}_{\mathbf{0}}=3$. The contrast enhancement image is inverted before the application of the wavelet transform to it, so that the vessels appear brighter than the background.

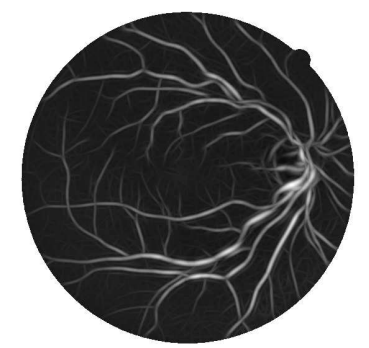

(a)

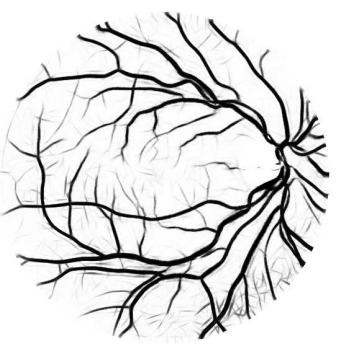

(b)
Figure 2: (a) Maximum modulus response of Gabor wavelet transform over 18 Gabor filters with scale value of $a=4$. (b) Segmentation of blood vessels.

The blood vessel segmentation is obtained using a Bayesian classifier with class conditional probability density functions, including a Gaussian mixture model, where each pixel is classified as a vessel or non-vessel pixel, as shown in Fig. 2(b). To reduce 
the computational burden, pattern matching is applied only to candidate pixels picked from the fundus image. The binary vessel/nonvessel image is thinned by applying a morphologic algorithm that reduces all objects in the binary image to the pixel dimension keeping in the new image only the central pixels of the vessels. The number of candidates is even more reduced by considering only the ones which are within an image area with $2.5 \%$ of the pixels with the highest intensity of the contracted image as showed in Fig. 3.

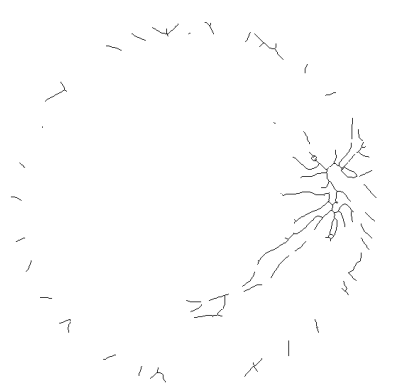

Figure 3: Thinned candidate vessels.

\subsection{Optic Disc Detection}

A distinguishing feature of the optic disc is that it is the region of convergence for the blood vessel network. The shape, colour, and size of the OD showed large variance especially in the presence of retinopathies, and therefore, detection methods based on these properties were shown to be weak, and impractical.

As stated by Hoover et al. (Hoover et al., 2000), "A matched filter describes the expected appearance of a desired signal, for purposes of comparative modeling". Thus, in order to detect the OD, a simple vessels' direction matched filter is proposed to roughly match the direction of the vessels at the OD vicinity. Based on the retinal vasculature orientations we define a $11 \times 11$ pattern formed by two opposite parables. The distance between the pattern and the thinned vessel map is obtained using the Earth Mover's distance (EMD) approach.

Rubner et al. (Rubner et al., 2000) introduced the EMD to measure perceptual similarity between images for the purpose of image retrieval. EMD evaluates dissimilarity between two distributions or signatures in some feature space where a distance measure is given. Intuitively, given two distributions, one can be seen as a mass of earth properly spread in space, the other as a collection of holes. Then, the EMD measures the least amount of work needed to fill the holes with earth. Here, a unit of work corresponds to transporting a unit of earth by a unit of ground distance. The EMD between two distributions is given

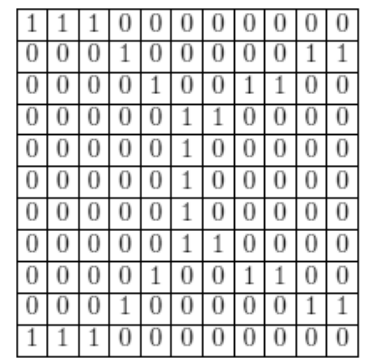

Figure 4: Matching pattern.

by the minimal sum of costs incurred to move all the individual points between the signatures.

Let $P=\left\{\left(p_{1}, w_{p_{1}}\right), \ldots,\left(p_{m}, w_{p_{m}}\right)\right\}$ be the first signature with $m$ pixels, where $p_{i}$ is the pixel representative and $w_{p_{i}}$ is the weight of the pixel; the second signature with $n$ pixels is represented by $Q=$ $\left\{\left(q_{1}, w_{q_{1}}\right), \ldots,\left(q_{n}, w_{q_{n}}\right)\right\}$; and $D=\left[d_{i j}\right]$ the distance matrix where $d_{i j}$ is the distance between two points' image coordinates $p_{i}$ and $q_{j}$. The flow $f_{i j}$ is the amount of weight moved from $p_{i}$ to $q_{j}$. The EMD is defined as the work normalized by the total flow $f_{i j}$, that minimizes the overall cost:

$$
\operatorname{EMD}(P, Q)=\frac{\sum_{i} \sum_{j} f_{i j} d_{i j}}{\sum_{i} \sum_{j} f_{i j}}
$$

As pointed by Rubner et al. (Rubner et al., 2000), if two weighted point sets have unequal total weights, EMD is not a true metric. It is desirable for robust matching to allow point sets with varying total weights and cardinalities. In order to embed two sets of contour features with different total weights, we divide the blood vessel image in several overlapping tiles and simulate equal weights by adding the appropriate number of points, to the lower weight set, with a penalty of maximal distance. As a measure of distance for the EMD, we use

$$
d_{i j}=1-e^{-\frac{\left\|p_{i}-q_{j}\right\|}{\alpha}}
$$

where $\left\|p_{i}-q_{j}\right\|$ is the Euclidean distance between $p_{i}$ and $q_{j}$ and $\alpha$ is used in order to accept some deformation of the matching pattern. The exponential map limits the effect of large distances, which otherwise dominate the result.

\section{RESULTS AND DISCUSSION}

The proposed method was tested with fundus images of the retina from the DRIVE database which contains 40 images (20 for training and 20 for testing). 


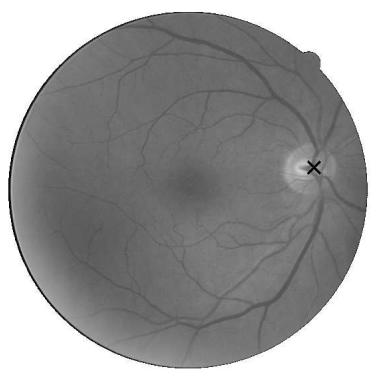

(a)

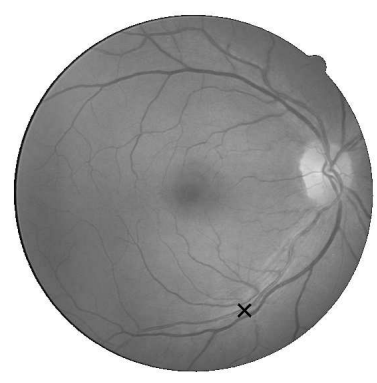

(e)

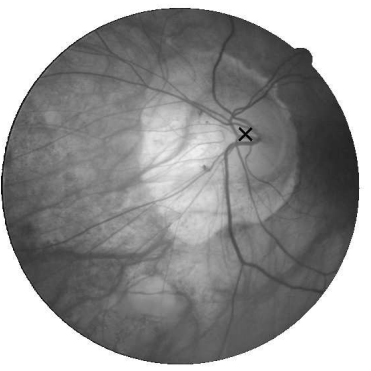

(b)

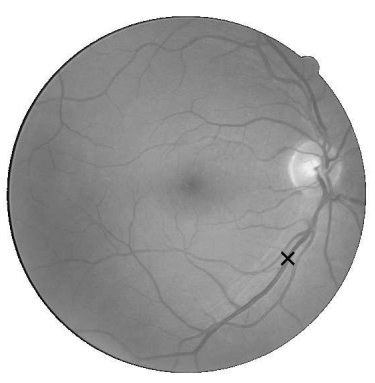

(f)

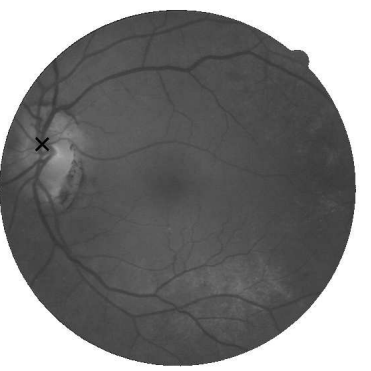

(c)

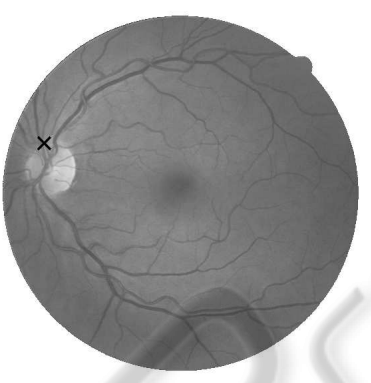

(g)

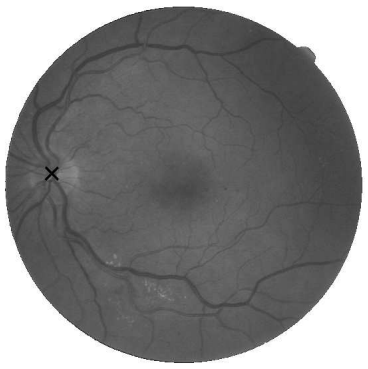

(d)

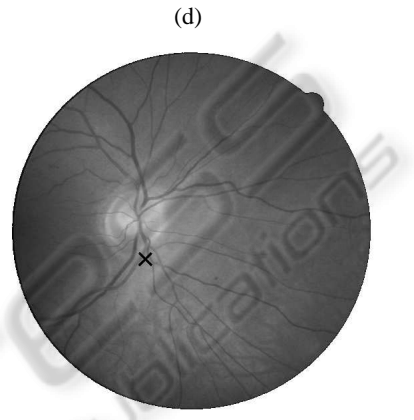

(h)

Figure 5: OD detection examples (black mark represents the estimated OD centre). The first row shows the best four results. The second row shows the worst four results. Images (e) and (b) of second row are the only two failed detections.

Figure 5 shows some illustrative OD detection results. First row shows the best four results and the second row shows the worst detections.

The proposed method achieved a success rate of 95\% (i.e., the OD was detected correctly in 38 out of the 40 images contained in the DRIVE database). The estimated centre is considered correct if it was positioned within 60 pixels of the manually identified centre, as proposed in (Foracchia et al., 2004),(Hoover et al., 2000),(Youssif et al., 2008). The average distance (for the 38 successful images) between the estimated OD centre and the manually identified center was 24.3 pixels. The only two cases in which the OD was not correctly detected (Images (e) and (f) of Fig. 5) were due to uneven crescent-shaped illumination joint with crossed vessels that biased the OD candidates and affected the vessel candidate selection.

\section{CONCLUSIONS}

In this paper, a simple and computationally efficient algorithm for automatic OD detection has been presented. The proposed algorithm takes advantage of the powerful preprocessing techniques such as the contrast enhancement, Gabor wavelet transform, mathematical morphology and Earth Mover's distance as the matching process. The performance of the proposed algorithm has been evaluated using
DRIVE database images. Good results have been achieved, failed the OD detection only in two of the forty images.

\section{REFERENCES}

Foracchia, M., Grisan, E., and Ruggeri, A. (2004). Detection of optic disc in retinal images by means of a geometrical model of vessel structure. IEEE Transactions on Medical Imaging, 23(10):1189-1195.

Hoover, A., Kouznetsova, V., and Goldbaum, M. (2000). Locating blood vessels in retinal images by piecewise threshold probing of a matched filter response. IEEE Transactions on Medical Imaging, 19(3):203-210.

Rubner, Y., Tomasi, C., and Guibas, L. (2000). The earth mover's distance as a metric for image retrieval. International Journal of Computer Vision, 40(2):99-121.

Soares, J., Leandro, J., Jr., R. C., Jelinek, H., and Cree, M. (2006). Retinal vessel segmentation using the 2$\mathrm{d}$ gabor wavelet and supervised classification. IEEE Transactions on Medical Imaging, 25(9):1214-1222.

Staal, J., Abramoff, M., Niemeijer, M., Viergever, M., and van Ginneken, B. (2004). Ridge-based vessel segmentation in color images of the retina. IEEE Transactions on Medical Imaging, 23(4):501-509.

Youssif, A., Ghalwash, A., and Ghoneim, A. (2008). Optic disc detection from normalized digital fundus images by means of a vessels' direction matched filter. IEEE Transactions on Medical Imaging, 27(1):11-18. 